\title{
As Implicações Formais do Uso de Diferentes Sistemas CAD e Prototipagem Rápida no Início do Processo de Projeto de Arquitetura
}

\author{
Formal Implications of Using Different Systems CAD and Rapid Prototyping in Early Architectural Design \\ Process
}

\author{
Larissa Milioli \\ Universidade de Brasília, Brasil \\ milioli@milioli.com.br \\ Neander Furtado Silva \\ Universidade de Brasília, Brasil \\ neander.furtado@gmail.com
}

\begin{abstract}
This article presents the formal results obtained from the same project, defined at the initial stage, modeled in three different CAD systems, exported and prototyped in two different types of three-dimensional printer. These digital systems have varying degrees of precision, can result in rapid prototyping different from the original virtual model, and this could frustrate the user's initial expectations, due to the need of modeling rework. The proper use of these tools can be helpful, since the beginning of the design process, with quick and reliable information, regarding the formal intent of the architect.
\end{abstract}

Keywords: Architectural design; CAD; Early process; Form; Rapid prototyping.

\section{Introdução}

A arquitetura pode ser interpretada como síntese entre forma, função e tecnologia condicionada ao tempo, custo e regulamentação. A forma da edificação emana das necessidades do usuário e dos materiais e técnicas disponíveis, levando em consideração o contexto urbano, possibilitando uma construção eficiente (Dijkstra,1985;2001 apud Voordt \& Wegen, 2013).

Neste contexto, as ferramentas de projeto e manufatura auxiliada por computador (CAD/CAM) apresentam vantagens como a redução do tempo de elaboração e maior precisão da representação do projeto. Os sistemas digitais de modelagem e prototipagem rápida foram, inicialmente, utilizados na arquitetura para representação final do projeto.

Atualmente, os sistemas de prototipagem rápida apresentam custos bastante reduzidos e com precisão satisfatória. Isto torna possível o uso desta tecnologia também em projetos de menor porte e para auxiliar o profissional desde o início do processo de projetação.

É necessário esclarecer que existem várias etapas a serem percorridas entre o modelo tridimensional virtual e o objeto fabricado. Orciuoli e Celani (2010) salientam que o modelo virtual precisa ser, na maioria dos casos, corrigido, adaptado e transformado em código de máquina para permitir sua compreensão pelos equipamentos de fabricação digital.
Por sua vez, os arquitetos, por não conhecerem adequadamente esses equipamentos, acabam não tirando proveito de suas possibilidades. Além disto, correm o risco de não obterem o modelo físico com os mesmos aspectos formais do modelo virtual projetado originalmente.

Este artigo apresenta os resultados formais obtidos em seis experimentos, de maneira que os sistemas digitais de modelagem e prototipagem rápida possam ser utilizados com menor retrabalho.

\section{Prototipagem Rápida Experimental}

A prototipagem rápida é um processo que utiliza a tecnologia CAD/CAM na produção de modelos físicos em escala reduzida, em diferentes tipos de materiais, antes que o artefato seja finalizado (Mitchell \& McCullough, 1995).

Os modelos físicos produzidos digitalmente, ou protótipos rápidos, podem ser descritos pela maneira como eles são produzidos e como podem ser usados. Schodek et al. (2005) definem como protótipos experimentais aqueles utilizados na concepção e desenvolvimento de projeto, e os protótipos pré-produção aqueles utilizados na execução final do trabalho. Isto determina o nível de precisão e tipo de material relacionado ao equipamento escolhido para a sua produção. 
Portanto, na etapa inicial do processo de projeto, um modelo prototipado com menor precisão ou resistência pode atender aos requisitos sem comprometer os aspectos formais essenciais do objeto final. Assim, estes sistemas devem oferecer rapidez e custo mais baixo na produção do modelo, sendo a técnica de fabricação digital por adição de camadas a mais utilizada.

Os protótipos rápidos experimentais permitem testar funcionalmente as opções e ideias, e ampliam os recursos de comunicação e interação, por meio da compreensão tátil entre os colaboradores, cliente e o próprio arquiteto.

\section{Formato STL}

Geralmente, os sistemas CAD tridimensionais incluem a opção de exportação no formato STL (Standard Tessellation Language), que por sua vez podem ser importados pela maioria dos softwares de impressoras tridimensionais. Este formato tem a vantagem de ser um padrão aberto.

Segundo Matias (2007), a geometria do modelo CAD em STL é descrita através de uma malha envolvente de triângulos. O arquivo contém o sistema tridimensional de coordenadas cartesianas dos nós de cada triângulo facetado, bem como a respectiva normal exterior da face.

A cor, textura ou outros atributos comuns ao modelo, não são representados no arquivo STL. O tamanho das faces é conduzido pela tolerância que controla a qualidade da representação da superfície, ou seja, a distância entre as faces dessa superfície.

\section{Experimentação}

No processo de experimentação verificamos se todos os elementos importados pelo sistema de prototipagem rápida produziram os modelos físicos digitais com a mesma representação geométrica formal do modelo CAD original.

O método de investigação adotado foi dividido nas seguintes etapas: CAD, Planejamento, Fabricação e Acabamento (Volpato et al., 2007 apud Pupo \& Celani, 2011).

Após a definição do projeto na etapa inicial, os seus elementos geométricos foram classificados. As medidas gerais do modelo CAD foram conferidas com base na largura, profundidade e altura de cada elemento arquitetônico externo, utilizando comandos específicos de cada software. O valor das medidas dos elementos equivalentes foi obtido diretamente do protótipo rápido com precisão em milímetros.

A forma do modelo também foi avaliada visualmente, de maneira que a representação dos elementos geométricos ortogonais deveria ser linear, constante e uniforme para aferir precisão.

No caso das geometrias curvas e superfícies complexas, o grau de resolução foi observado pela representação em maior ou menor número de faces em elementos planos que podem variar em seu tamanho e padrão triangular.

\section{Primeira Fase: Modelagem no sistema CAD}

A modelagem do projeto em sistema CAD foi a primeira fase do processo de experimentação. Para obtenção deste modelo de maneira suficientemente abrangente, foram utilizados três tipos diferentes de sistemas CAD, que oferecem ferramentas apropriadas para modelagem e documentação do projeto.

Os dois sistemas CAD genéricos utilizados foram o AutoCAD 2012 (Autodesk) e o Formz 6.7.3 (AutoDesSys). Foi utilizado também um sistema CAD específico (BIM), o Revit Architecture 2012 (Autodesk).

O projeto arquitetônico foi definido diretamente no ambiente gráfico do sistema CAD específico, obtendo-se o primeiro modelo nomeado como modelo CAD (A).

Um programa de necessidades de escritório de arquitetura foi préestabelecido para o mesmo: área de trabalho para cinco pessoas, um banheiro, uma copa, recepção e uma sala de reunião no mezanino. A edificação era de pequeno porte com área máxima de $250 \mathrm{~m}^{2}$. O projeto destinava-se a um terreno existente, visando considerar as condicionantes visuais, topográficas, de insolação e de ventilação.

O projeto apresenta uma volumetria compondo formas retilíneas simples e formas curvas complexas, de maneira a justificar o uso do sistema de prototipagem rápida. Ao mesmo tempo deveria possibilitar fácil entendimento para a sua reprodução em diferentes sistemas CAD.

Para uma melhor delimitação do grau de detalhamento a ser considerado na modelagem, adotaram os mesmos aspectos formais de maquetes preliminares. Assim, a preocupação era oferecer recurso para visualização do espaço e da volumetria.

O modelo foi planejado como elemento único, produzido sem a necessidade de montagem de partes separadas. Os elementos transparentes e aqueles utilizados para efeito de humanização, como o mobiliário, vegetação, pessoas e terreno não foram considerados para prototipagem.

$\mathrm{Na}$ modelagem dos elementos geométricos curvos e superfícies complexas foram adotadas as configurações, com resolução default, de cada sistema CAD utilizado. Isto porque, nesta etapa de projeto, as informações mais detalhadas da forma ainda não haviam sido confirmadas.

Após o término da definição do modelo CAD A (Revit), obteve-se os desenhos e medidas necessários para modelar o mesmo projeto nos outros dois sistemas $C A D$ genéricos, o modelo $C A D B$ (AutoCAD) e o modelo CAD C (FormZ).

Os comandos de edição das superfícies complexas do modelo CAD A não ficaram disponíveis para eventuais alterações. Esta limitação não ocorreu nos modelos $B$ e $C$ obtidos nos sistemas CAD genéricos.

Algumas medidas preventivas foram adotadas, no sentido de reduzir problemas na transferência de dados exportados do 
modelo CAD, como construir cada tipo de elemento geométrico fechado ou unido. No caso dos sistemas CAD genéricos, adotou-se também a construção do modelo virtual nos quadrantes positivos em $x, y$ e $z$.

A versão 2012 do Revit Architecture não possui originalmente o comando STLExport. Foi necessário importar este comando na opção Add-Ins>External Tools, para exportar o modelo no formato STL.

Entre os softwares CAD escolhidos para elaborar o experimento, somente o FormZ permitiu, além de exportar, importar o arquivo STL. Este fato viabilizou avaliar da representação geométrica dos arquivos STL gerados, e obter o número de faces produzidas em cada um dos três modelos CAD.

\section{Segunda Fase: Planejamento}

\section{Terceira Fase: Fabricação no Sistema de Prototipagem Rápida}

Na fase de fabricação foi muito importante seguir as instruções do manual de equipamento do fabricante. Os arquivos no formato padrão de cada impressora tridimensional foram abertos, um de cada vez, no software do respectivo equipamento, e verificado o tempo gasto estimado para produzir cada protótipo. Um total de seis protótipos rápidos foi produzido, sendo os (AZ), (BZ) e (CZ) pela impressora Zprinter, e (AX), (BX) e (CX) pela impressora ThingO-Matic.

\section{Quarta Fase: Acabamento}

$\mathrm{Na}$ quarta e última fase do experimento, foram realizados os procedimentos de limpeza e de resinagem com o material "Z-Bond 101" nos protótipos rápidos (AZ), (BZ) e (CZ), fabricados pela Zprinter. Os protótipos rápidos $(A X),(B X)$ e $(C X)$, fabricados pela impressora Thing-O-Matic, tiveram alguns pontos lixados, por conta de excesso de material.

A preocupação na fase do acabamento foi a de não alterar a geometria do protótipo, para não interferir na avaliação dos resultados deste experimento.

\section{Análise dos Resultados}

Os resultados foram bastante homogêneos e as pequenas diferenças ocorreram na resolução das curvas e superfícies complexas dos protótipos, que por sua vez, são reproduzidas de maneira idêntica ao modelo virtual do arquivo formato STL importado no software de prototipagem.

Estes elementos geométricos são representados e reproduzidos facetados, e não como a imagem renderizada do modelo CAD, onde as superfícies são representadas com a resolução de faces curvas.

Este fato não foi observado no protótipo $A Z$, produzido pela Um dos sistemas definidos para prototipagem rápida foi a impressora tridimensional ZPrinter 310 Plus, da ZCorporation, que utiliza o método de adição de camadas, com material em pó "zp 150" e aglutinante "zb60 clear". O software ZPrint 7.10 é fornecido com o equipamento para importar, configurar e enviar o arquivo para produzir o protótipo digitalmente.

O outro sistema de prototipagem adotado foi a impressora ThingO-Matic, da MakerBot. Esta utiliza plástico ABS com filamento de espessura igual a 0,175 $\mathrm{mm}$. Este sistema emprega o método aditivo por deposição e fusão (FDM). O software disponibilizado pelo fabricante foi o ReplicatorG 29.

Os arquivos STL gerados nos sistemas CAD foram importados um a um, pelo software de cada impressora tridimensional, possibilitando verificar que todos os elementos geométricos de cada modelo estavam representados integralmente.

No caso de ocorrer qualquer problema de representação no modelo, recomenda-se efetuar a correção no sistema CAD original.

Nesta fase foi possível definir as configurações para impressão do modelo, como o ponto de origem e a rotação. A escala adotada para a produção dos protótipos da impressora Zprinter foi 1:100, e da impressora Thing-O-Matic 1:200, devido à menor base de impressão desta. É importante conferir o limite de espessura do equipamento, conforme a relação da medida do elemento modelado e a escala definida.

Portanto, foram gerados seis arquivos com as configurações finais: três a partir da impressora Zprinter; e três arquivos a partir da impressora Thing-O-Matic.

impressora Zprinter, cuja representação da superfície complexa foi produzida sem faces planas aparentes, semelhantemente ao modelo virtual original. A causa provável é a resolução maior da configuração default, do sistema CAD utilizado. Evidentemente este sistema CAD exportou o arquivo formato STL com tamanho muito maior, comparado com os arquivos exportados dos outros sistemas.

Tabela 1: Tamanho dos arquivos dos modelos CAD, formato STL e números de faces.

\begin{tabular}{|c|c|c|c|}
\hline $\begin{array}{c}\text { Formato } \\
\text { Arquivo }\end{array}$ & $\begin{array}{c}\text { Modelo A } \\
\text { (Revit) }\end{array}$ & $\begin{array}{c}\text { Modelo B } \\
\text { (Autocad) }\end{array}$ & $\begin{array}{c}\text { Modelo C } \\
\text { (Formz) }\end{array}$ \\
\hline Modelo CAD & $26.696 \mathrm{~KB}$ & $318 \mathrm{~KB}$ & $672 \mathrm{~KB}$ \\
\hline Arquivo STL & $1.560 \mathrm{~KB}$ & $69 \mathrm{~KB}$ & $69 \mathrm{~KB}$ \\
\hline № Faces & 6.340 & 1.402 & 1.398 \\
\hline
\end{tabular}

Os sistemas CAD genéricos produziram arquivos STL com tamanhos e número de faces muito similares entre si. Mesmo assim, a resolução foi diferenciada quanto ao tamanho e tipo de representação das faces das superfícies complexas no arquivo STL exportado.

Os três protótipos rápidos produzidos pela impressora Thing-OMatic (AX, BX e CX) apresentaram falha semelhante na representação das vigas externas frontais, com altura e 
forma irregular. Isto provavelmente decorre da precisão limitada do equipamento e do processo construtivo utilizado, como base

Tabela 2: Modelos virtuais (MV) e protótipos rápidos (PR) obtidos. para produção das dimensões mínimas do perfil deste elemento geométrico.

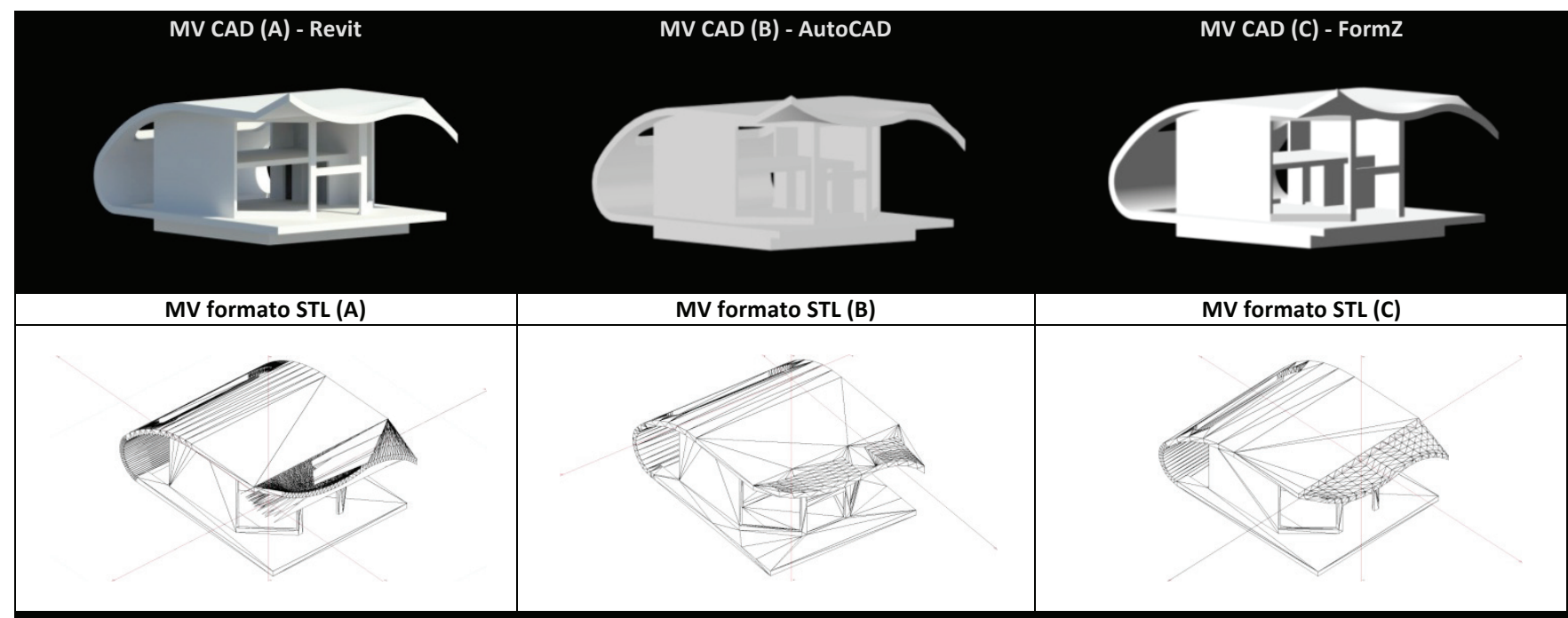

PR Thing-O-Matic (AX)

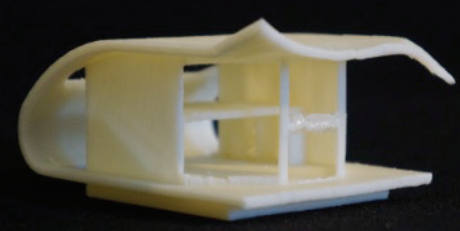

PR Zprinter (AZ)

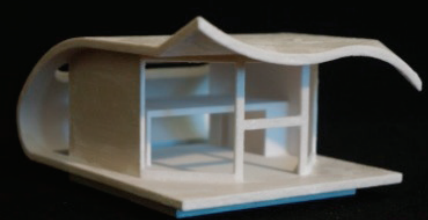

PR Thing-O-Matic (BX)

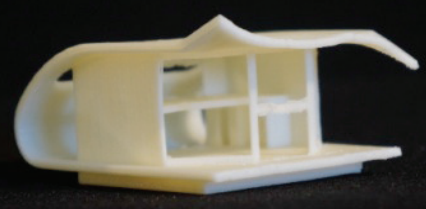

PR Zprinter (BZ)

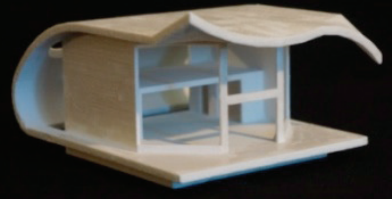

PR Thing-O-Matic (CX)

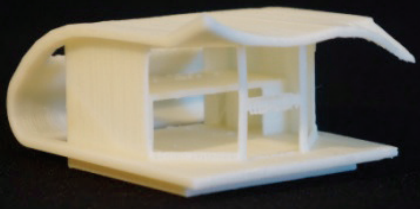

PR Zprinter (CZ)

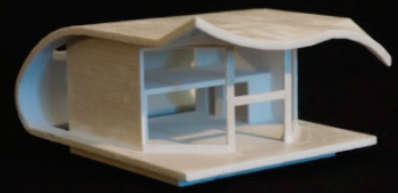

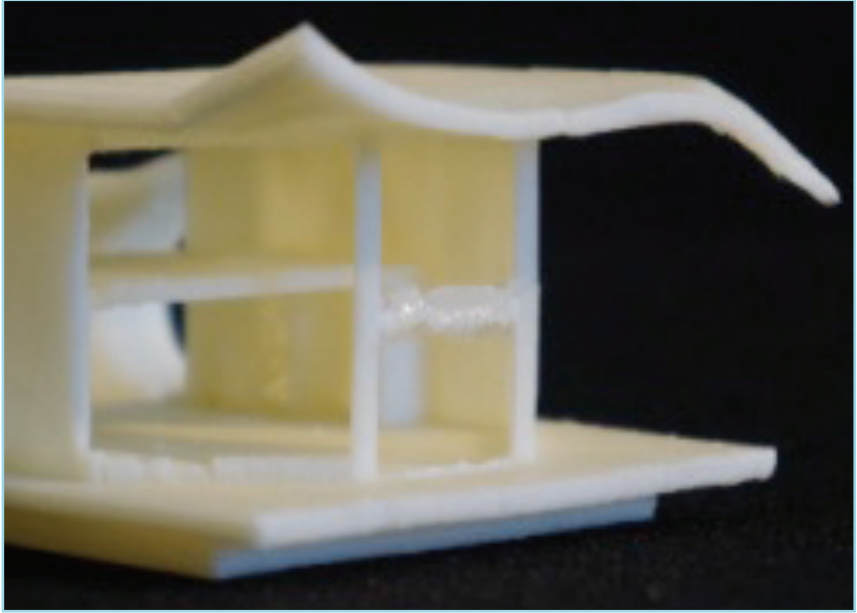

Figura 1: Falhas na representação das vigas frontais do protótipo rápido $A X$ (Thing-O-Matic) comparado ao modelo CAD A (Revit).

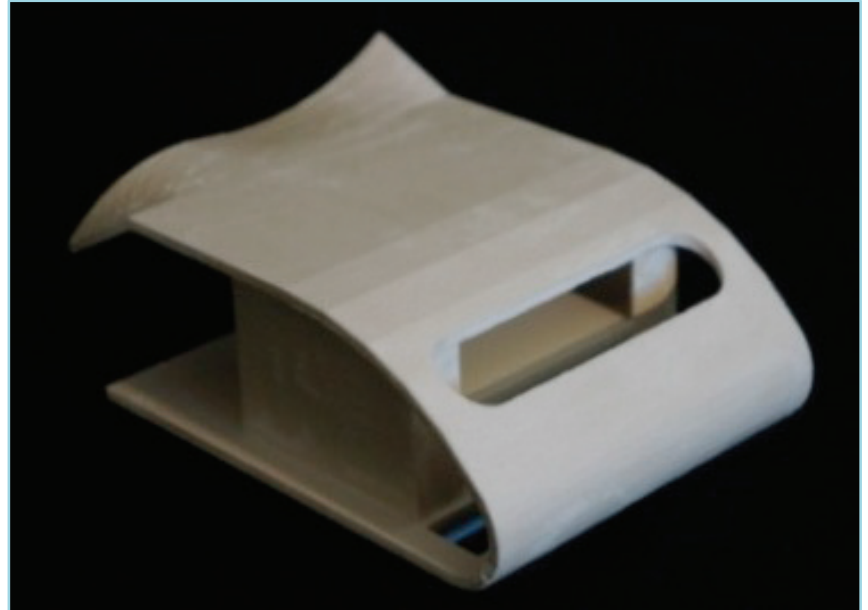

Figura 2: Superfícies curvas e complexas com representação facetada do protótipo rápido CZ (Zprinter) comparado ao modelo CAD C (FormZ). 


\section{Conclusão}

Um mesmo projeto arquitetônico, modelado em diferentes sistemas CAD, pode apresentar diferentes graus de resolução na representação de superfícies curvas e complexas, quando importadas e produzidas a partir de um mesmo sistema de prototipagem rápida.

Portanto, os protótipos resultantes não foram exatamente iguais para todos os sistemas utilizados, devido aos diferentes níveis de resolução dos arquivos STL gerados para a transferência de dados, e às limitações de precisão dos próprios sistemas de prototipagem.

As informações obtidas nos experimentos sugerem que no momento da modelagem a resolução dos elementos com superfícies curvas seja aumentada ao máximo possível visando produzir formas sem aspecto facetado. A vantagem é que no processo de fabricação por adição de camadas, o arquivo STL gerado não aumenta o tempo de fabricação do modelo prototipado.

Se a intenção for tirar partido da resolução facetada das superfícies curvas, sugere-se manter a resolução baixa desses elementos geométricos ou ainda mantê-los na resolução default fornecida pelo sistema CAD.

O resultado do aspecto formal pode ser previamente visualizado na fase de planejamento, sem a necessidade de produção do modelo.

A obtenção de um protótipo rápido idêntico ao modelo virtual original depende tanto da resolução definida no sistema CAD, observadas nas superfícies curvas e complexas, quanto do grau de precisão oferecido pelo equipamento de prototipagem rápida, responsáveis diretamente pela precisão em temos de dimensionamento dos elementos geométricos.

Os resultados obtidos sugerem que a modelagem em diferentes sistemas CAD e de prototipagem rápida, desde o início do processo de projetação, pode ser considerada um recurso confiável, pelo fato de oferecer ferramentas capazes de representar de maneira rápida e fidedigna a intenção formal do arquiteto.

\section{Referências}

Matias, J. M. (2007). Simulação e Optimização de Processos Estereolitográficos. Dissertação de Mestrado. Universidade de Aveiro. Departamento de Engenharia Mecânica. (141 p.).

Mitchell, W. J., \& McCullough, M. (1995). Digital Design Media. New York: Van Nostrand Reinhold.

Orciuoli, A., \& Celani, G. (2010, Dezembro). Interseção - 3D em Detalhes. Pini. Revista AU, 201 (25), 68-71.

Pupo, R., \& Celani, M. G. C. (2011). Prototipagem rápida e fabricação digital na Arquitetura: fundamentação e formação. In: Kowaltowski, D. C. C. K., Moreira, D. de C., Petreche, J. R. D., \& Fabrício, M. M. (Org.). O Processo de Projeto em Arquitetura da Teoria à Tecnologia. São Paulo: Oficina de Textos.

Schodek, D., Bechthold, M., Griggs, K., Kao, K. M., \& Steinberg, M. (2005). Digital Design and Manufacturing: CAD/CAM Applications in Architecture and Design. New Jersey: John Wiley \& Sons.

Voordt, T. J. M. van der, \& Wegen, H. B. R. van. (2013). Arquitetura sob o olhar do usuário: programa de necessidades, projeto e avaliação de edificações. Tradução Maria Beatriz de Medina. São Paulo: Oficina de Textos. 